\section{Imaging prostate cancer metastasis}

\section{By Brian Moy, Staff Writer}

Determining the status of lymph node metastasis in prostate cancer is important for deciding treatment options, but current techniques for detecting metastasis are imprecise and invasive. A paper published in Nature Medicine describes a noninvasive imaging method that uses recombinant human adenoviral vectors to directly detect sentinel lymph node metastasis.

Sentinel lymph nodes are typically the first draining lymph nodes to which cancer cells metastasize. Importantly, the adenoviral vectors have an innate affinity for the lymphatic system. However, applying the technique to humans may be challenging because it could be difficult to control where the vector goes once it is injected into the body.

If the technological kinks are worked out, there is potential for coupling the imaging technology with gene therapy to simultaneously detect and treat metastatic prostate cancer.

Researchers at the University of California, Los Angeles (UCLA) engineered prostate cancer-specific viral vectors with imaging reporter genes whose expression could be visualized using PET.

The researchers designed a lentivirus that expressed the reporter gene herpes simplex virus thymidine kinase (sr39tk) under the control of a prostate-specific antigen (PSA) promoter system known as the twostep transcriptional activation (TSTA) system. The TSTA system was engineered to confer high expression of the reporter genes when in the presence of targeted prostate cancer cells and PSA.

In a mouse model of prostate cancer, peritumoral administration of the lentivirus in conjunction with its tracer $9-\left(4\left[{ }^{18} \mathrm{~F}\right]\right.$ fluoro-3-hydroxymethylbutyl) guanine $\left({ }^{18} \mathrm{FHBG}\right)$ resulted in the direct detection of tumor growth and sentinel lymph node metastasis using PET. ${ }^{1}$

Lily $\mathrm{Wu}$, principal investigator and corresponding author of the Nature Medicine paper, said that "because lymph nodes in prostate cancer are so difficult to access for biopsy, the advantage of the study is that the vectors provide a noninvasive alternative for detecting metastasis." Wu is an associate professor in the Department of Molecular and Medical Pharmacology and in the Department of Urology at the UCLA School of Medicine.

Although other noninvasive techniques such as MRI and computed tomography $(\mathrm{CT})$ scan are available, neither is able to differentiate between swollen lymph nodes caused by inflammation or by deposition of malignant cells. ${ }^{2}$

Thus, said Wael Yared, chief technology officer at VisEn Medical Inc., molecular imaging can expand the capabilities of anatomical imaging techniques such as MRI and CT scan by allowing for the "earlier detection of molecular alterations that signal the presence of cancer at a still curable stage."

\section{Gene therapy pairing}

The UCLA researchers think the natural fit for their imaging technology is to pair it with gene therapies. The coupling, they said, "would enable not only the accurate assessment of lymph node status, but also the prospect of preventing metastatic outgrowth and systemic spread."

$\mathrm{Wu}$ and colleagues plan to conduct preclinical testing of imaging coupled with gene therapy to treat prostate cancer in dogs.

Wu said a single vector would be able to deliver genes for both killing and imaging cancer cells. In some cases, one gene can accomplish both tasks. For example, in addition to functioning as a PET-imaging reporter gene, sr39tk also functions as a suicide gene in the presence of the prodrug ganciclovir. ${ }^{3}$

Jason Alter, director of marketing at Aureon Laboratories Inc., said the study has promise for detecting and imaging advanced disease. He added that "in the future, the imaging method may be important for being able to better stage advanced prostate cancer to subsequently improve care and treatment for these patients. Although a ways away, imaging coupled with gene therapy has exciting potential."

Aureon markets its Prostate $\mathrm{Px}+$ test to predict prostate cancer progression and disease recurrence at the time of diagnosis. Using a patient's original biopsy, Prostate $\mathrm{Px}+$ integrates a spatial analysis of tissue histology, measurements of molecular biomarkers associated with disease progression and clinical data provided by a physician to determine the likelihood of metastasis and disease progression.

John Babich, president and CSO of Molecular Insight Pharmaceuticals Inc., told SciBX that the method published in Nature Medicine "could be useful if you are going after prostate cancer from a gene therapy perspective because you can add an imaging reporter gene that would tell you if the therapy actually hit your target."

Molecular Insight is conducting a Phase I trial of a pair of radiolabeled prostate-specific membrane antigen (PSMA)-targeted molecular imaging agents, MIP-1072 and MIP-1095, to diagnose recurrent metastatic prostate cancer. The company plans to select a lead candidate based on the results of the trial and develop it as Trofex.

Babich did note that it is hard to control where a vector goes once injected into the body. "There is no reason for viral vectors to only target the tumor," he said.

Babich suggested that delivery of traceable viral vectors carrying therapeutic genes would be most useful in cases of localized disease before metastasis has occurred. 
Michael Manyak, VP of medical affairs at EUSA Pharma Inc., agreed. One problem, he noted, is that "the vectors are lymph node specific, not tumor specific," and i.v. administration of the vector in humans would result in the targeting of lymph nodes throughout the body.

To circumvent this problem, Manyak pointed to one strategy that was employed by the investigators in the study: directly injecting the vectors into the tumors. However, he said, this is not always feasible in humans because the tumor may not always be easily accessible for injection.

Manyak added that the aggressive tumor model used in the study does not mirror what generally happens in prostate cancer as disease progression is usually slow. "The fact that the researchers were able to use PET scanning in the study is directly correlated to the fact that they used highly aggressive tumors. PET scans with FDG are not very useful in prostate cancer."

Fluorodeoxyglucose (FDG) is a biologically active glucose analog commonly used in PET scans to introduce a radionuclide tracer into the body.

EUSA markets ProstaScint III-indium capromab pendetide, a radiolabeled $\mathrm{mAb}$ that recognizes PSMA, to image the extent and spread of prostate cancer.

\section{REFERENCES}

1. Burton, J. et al. Nat. Med.; published online July 11, 2008; doi: $10.1038 / \mathrm{nm} .1727$

Contact: Lily Wu, University of California, Los Angeles, Calif. e-mail: Iwu@mednet.ucla.edu

2. Rorvik, J. \& Haukaas, S. Curr. Opin. Urol. 11, 181-188 (2001)

3. Rosenfeld, M.E. et al. Clin. Cancer Res. 1, 1571-1580 (1995)

COMPANIES AND INSTITUTIONS MENTIONED

Aureon Laboratories Inc., Yonkers, N.Y.

EUSA Pharma Inc., King of Prussia, $\mathrm{Pa}$.

Molecular Insight Pharmaceuticals Inc. (NASDAQ:MIPI), Cambridge, Mass.

University of California, Los Angeles, Calif.

VisEn Medical Inc., Woburn, Mass. 